\title{
Prevalência de cárie radicular e condição periodontal em uma população idosa institucionalizada de Piracicaba - SP
}

\section{Prevalence of root caries and periodontal conditions in an elderly institutionalized population from Piracicaba - SP}

\author{
Marcelo de Castro MENEGHIM* \\ Antonio Carlos PEREIRA* \\ Fábio Rodrigo Biasin SILVA**
}

\begin{abstract}
MENEGHIM, M. de C.; PEREIRA, A. C.; SILVA, F. R. B. Prevalência de cárie radicular e condição periodontal em uma população idosa institucionalizada de Piracicaba - SP. Pesqui Odontol Bras, v. 16, n. 1, p. 50-56, jan./mar. 2002.

A evolução dos procedimentos preventivos e curativos em Odontologia, verificados nas duas últimas décadas, levou a uma maior longevidade dos elementos dentários, acarretando com isso, uma maior demanda por tratamento periodontal e, em especial, cárie radicular no paciente idoso. O presente estudo teve como objetivo verificar as condições periodontais e lesões radiculares, de uma amostra de população institucionalizada do município de Piracicaba - SP. Nesse estudo, foram examinados 151 idosos com idade entre 54 e 93 anos. Os sextantes foram examinados e classificados do ponto de vista de saúde periodontal, necessidade de tratamento periodontal, presença de retração gengival e lesões radiculares, utilizando-se como critérios os indicadores da OMS (1999). Comparando-se duas faixas etárias: de pacientes com idade entre 50 e 75 anos e pacientes com idade acima de 75 anos, constatou-se que há um aumento na prevalência de lesões de cárie e outras afecções radiculares, como erosão e abrasão, indicando aumento na susceptibilidade a tais lesões com o passar da idade.
\end{abstract}

UNITERMOS: Odontologia geriátrica; Epidemiologia; Cárie radicular.

\section{INTRODUÇÃO}

De acordo com o relatório publicado pela ONU (Organização das Nações Unidas) sobre o Índice de Desenvolvimento Humano - 1998, o Brasil chega, pela primeira vez, ao grupo dos países com alto nível de desenvolvimento humano. Este índice refere-se a indicadores de três áreas: Saúde, Educação e Renda, colocando o Brasil na $62^{a}$ posição, entre 174 países $^{17}$. No período entre 1994 e 1995, o indicador utilizado pelo Programa das Nações Unidas para o Desenvolvimento, na área de Saúde, é a esperança de vida ao nascer e, nesse caso, a expectativa de vida para os nascidos em 1995 seria de 66,6 anos.

O aumento da expectativa de vida da população brasileira, registrado nos últimos anos, tem trazido para o País uma situação nova, com uma porcentagem cada vez maior das faixas etárias de maior idade. De acordo com o IBGE, em 1960, a faixa de 60 anos era responsável por 4,9\% do total da população brasileira e, em 1999, já atingiu 8,7\%, uma das maiores do mundo em números absolutos $^{6}$.

Em virtude do declinio da ocorrência de cáries coronárias, aumenta a perspectiva de um maior número de dentes com desenvolvimento de doença periodontal e exposição radicular, presentes na boca. Tal situação justifica a necessidade de pesquisas direcionadas à população idosa, que deve, inclusive, passar a receber maiores recursos, que até então eram destinados exclusivamente ao estudo de cáries coronárias em crianças ${ }^{7,11,19}$.

Diante do quadro social e dos motivos anteriormente expostos, justifica-se a importância do estudo epidemiológico das condições bucais da população idosa, entre outros, para o desenvolvimento de programas odontológicos mais específicos para o paciente idoso.

Mediante isso, o presente estudo teve como objetivo verificar as condições periodontais e lesões radiculares de uma amostra de população institucionalizada do município de Piracicaba - SP. 
MENEGHIM, M. de C.; PEREIRA, A. C.; SILVA, F. R. B. Prevalência de cárie radicular e condição periodontal em uma população idosa institucionalizada de Piracicaba - SP. Pesqui Odontol Bras, v. 16, n. 1, p. 50-56, jan./mar. 2002.

\section{MATERIAL E MÉTODO}

\section{Termo de informação e consentimento para participação em pesquisa}

De acordo com a Resolução 196 de 1996 do Conselho Nacional de Saúde do Ministério da Saúde, referente à participação de humanos em pesquisa clínica, foi entregue um Termo de Consentimento de Pesquisas em Humanos para cada voluntário.

\section{Amostra}

Este estudo foi realizado com 151 indivíduos pertencentes a uma população institucionalizada ("Lar dos Velhinhos" na cidade de Piracicaba - SP) de ambos os sexos e idade variando entre 53 e 94 anos conforme a Tabela 1. Para a tabulação dos resultados, os idosos foram divididos em dois grupos, o primeiro formado por idosos de ambos os sexos com idade entre 50 e 75 anos de idade e, o segundo grupo, com idosos acima de 75 anos. A percentagem do sexo feminino foi maior em ambas as faixas etárias, sendo mais acentuada na faixa etária com idade acima de 75 anos. No total, a percentagem foi de $60 \%$ para o sexo feminino e $40 \%$ para o sexo masculino.

Não foram incluídas na amostra as pessoas que apresentavam: dentista particular fixo, recusa ao exame e aquelas com alterações de saúde ou que não estivessem sob tratamento médico controlado e que em conseqüência pudesse interferir no resultado do levantamento ${ }^{13}$.

\section{Forma de realização dos exames}

Os exames foram realizados por um único examinador, com o paciente sentado, em cadeiras comuns, cadeira de rodas ou camas, sob luz natural, utilizando-se apenas de espelho bucal plano, sonda exploradora $n^{\circ} 5$, sonda periodontal tipo OMS e secagem prévia das superficies dentais com gaze estéril. Não foram utilizadas radiografias. Os resultados foram anotados em fichas especialmente desenvolvidas para este estudo.

\section{Calibração}

O processo de calibração ${ }^{14}$ foi desenvolvido de acordo com as seguintes etapas: a) teórico: critérios, códigos, técnica de exame; b) prática: exame clínico, apuração e análise dos resultados. A calibração foi desenvolvida num total de 20 horas.

O erro intra-examinador, medido pela estatística Kappa, foi satisfatório $(K>0,75)$. Para a manutenção dos critérios de diagnóstico, foram realizados exames em duplicata, em cerca de $10 \%$ da amostra do levantamento principal ${ }^{14}$.

\section{Forma de análise dos resultados}

A análise estatística realizada foi descritiva, com apresentação tabular dos dados clínicos. Os dados coletados foram processados, utilizando-se o software Excell.

\section{Critérios adotados}

\section{a) Condição periodontal}

Para a avaliação periodontal, foi utilizado o índice CPITN (Índice Periodontal Comunitário de Tratamento de Necessidades) proposto pela $\mathrm{OMS}^{14}$, assim descrito: $\mathrm{X}$ - sextante excluído, 0- sextante hígido, 1- sextante com presença de sangramento em pelo menos um dos dentes, 2- presença de cálculo supra- ou subgengival, 3- bolsa periodontal de 4 a $5 \mathrm{~mm}$ e 4- bolsa periodontal com $6 \mathrm{~mm}$ ou mais profunda. Para a determinação da necessidade de tratamento em função da severidade da doença periodontal, utilizou-se a seguinte classificação ${ }^{1}$ : 0- (nenhum tratamento - código 0), I(instrução de higiene bucal - código 1), II- (I + instrumentação periodontal - códigos 2 e 3), III- (I + II + tratamento complexo - código 4).

Utilizou-se, para o exame, de uma sonda periodontal que apresenta em sua ponta uma esfera de $0,5 \mathrm{~mm}$ de diâmetro e apresenta uma área demarcada em preto, situada entre $3,5 \mathrm{~mm}$ e $5,5 \mathrm{~mm}$ da ponta da esfera.

A boca é dividida em sextantes definidos pelos números dos dentes 18-14, 13-23, 24-28, 38-34, 33-43, 44-48. Um sextante só será examinado se

TABELA 1 - Número de pacientes examinados por faixa etária e sexo. Piracicaba, 1998.

\begin{tabular}{l|c|c|c|c|c|c}
\hline \multirow{2}{*}{ Idade } & \multicolumn{2}{|c|}{ Masculino } & \multicolumn{2}{c}{ Feminino } & \multicolumn{2}{c}{ Total } \\
\cline { 2 - 7 } & $\mathrm{n}$ & $\%$ & $\mathrm{n}$ & $\%$ & $\mathrm{n}$ & 81 \\
\hline 50-75 anos & 37 & 46 & 44 & 54 & 70 & 100 \\
\hline Mais de 75 anos & 23 & 33 & 47 & 60 & 151 & 100 \\
\hline Total & 60 & 40 & 91 & 67 & & 100 \\
\hline \hline
\end{tabular}


MENEGHIM, M. de C.; PEREIRA, A. C.; SILVA, F. R. B. Prevalência de cárie radicular e condição periodontal em uma população idosa institucionalizada de Piracicaba - SP. Pesqui Odontol Bras, v. 16, n. 1, p. 50-56, jan./mar. 2002.

existir dois ou mais dentes presentes e não indicados para extração.

Os dentes índices são: 17-16, 11, 26-27, 37-36, 31, 46-47. Em caso de ausência desses dentes, serão utilizados os outros dentes do sextante. O sextante será classificado baseando-se na pior situação encontrada. Cada dente deve ter seis pontos de sondagem: mésio-vestibular, médio-vestibular, disto-vestibular, mésio-lingual, médio-lingual, disto-lingual.

Para avaliar a retração do tecido gengival, utilizou-se de sonda periodontal tipo OMS, verificando-se a distância do tecido gengival em relação à junção amelocementária (JAC). Para todos os elementos dentais presentes na boca, esta retração foi quantificada (em $\mathrm{mm}$ ) e apenas a superficie dental de maior retração foi considerada. Para uma distância entre 0 e $0,5 \mathrm{~mm}$ do tecido gengival em relação à JAC, considerou-se ausência de retração gengival (código 0). Para os códigos de I a IV, a presença de retração gengival foi considerada da seguinte forma: I- entre 0,5-3 $\mathrm{mm}$, II- 3-5 mm, III5-7 $\mathrm{mm}$ e IV- maior que $7 \mathrm{~mm}$.

\section{b) Condição radicular}

A presença de lesões radiculares foi verificada através da utilização de espelho bucal plano e sonda exploradora $\mathrm{n}^{\circ} 5$ após limpeza e secagem da superfície dental com gaze estéril.

A diferenciação de lesões ativas de lesões inativas de cárie realizou-se através da constatação da presença ou ausência de manchas esbranquiçadas e/ou tecido amolecido, seguindo a seguinte classificação: 0- ausência de lesão, L1- lesão inativa de cárie radicular, L2- lesão ativa, L3- obturação e L4- erosão/abrasão. O critério obturado foi considerado quando observada a presença de restaurações situadas exclusivamente abaixo da JAC. Quando as restaurações encontradas iniciaram na coroa e estenderam-se mais de $0,5 \mathrm{~mm}$ abaixo da $\mathrm{JAC}$, foram classificadas como restauradas.

\section{RESULTADOS}

Analisando-se a Tabela 2, em relação à faixa etária de 50 a 75 anos de idade, nota-se que 90,5\% dos indivíduos apresentaram menos de dois dentes por sextante, resultando numa média de 5,44 sextantes por indivíduo, e, dessa forma, foram excluídos da análise. Em relação à faixa etária de mais de 75 anos, observou-se um aumento no número de sextantes excluídos, 5,82 sextantes por indivíduo, cerca de 97,2\% do total nessa idade.

Assim sendo, para a faixa etária de 50 a 75 anos de idade, 0,56 sextantes puderam ser classificados como válidos e, para a faixa etária de 75 anos ou mais, apenas 0,18 sextante por indivíduo, puderam ser classificados desta maneira (Tabela 2).

Do total de sextantes válidos, a faixa etária de 50 a 75 anos apresentou 0,17 sextantes, ou $30,4 \%$ classificados como hígidos. Do restante dos sextantes válidos que representam $69,6 \%, 19,7 \%$ foram classificados com o código 1 , ou seja, apresentavam sangramento gengival quando submetido à sondagem; $32,1 \%$ dos sextantes, código 2 , contendo cálculo aderido à superficie dental e 17,8\%, classificados com o código 3 ou 4, sextantes com dentes que apresentavam bolsa periodontal, conforme Tabela 3 .

Dos sextantes válidos, para a faixa etária de mais de 75 anos, 0,12 sextantes ou $66,7 \%$, foram classificados como hígidos; 0,04 sextantes ou

TABELA 2 - Média, porcentagem e número de sextantes afetados, por indivíduo, em função do estado periodontal, segundo a faixa etária. Piracicaba, 1998.

\begin{tabular}{c|c|c|c|c|c|c}
\hline \hline \multirow{2}{*}{ Estado periodontal } & \multicolumn{3}{|c|}{$50-75$ anos } & \multicolumn{3}{c}{ Mais de 75 anos } \\
\cline { 2 - 7 } & Média & $\%$ & Sextante/indivíduo & Média & Sextante/indivíduo \\
\hline Sextante excluído & 73,3 & 90,5 & 5,44 & 68,0 & 97,2 & 5,82 \\
\hline 0 & 2,3 & 2,9 & 0,17 & 1,5 & 2,1 & 0,12 \\
\hline 1 & 1,5 & 1,9 & 0,11 & 0,3 & 0,5 & 0,2 \\
\hline 2 & 2,5 & 3,1 & 0,18 & 0,2 & 0,04 & 0,02 \\
\hline 3 & 0,8 & 1,0 & 0,06 & 0,0 & 0,00 \\
\hline 4 & 0,5 & 0,6 & 0,04 & 0,0 & 0,0 & 0,00 \\
\hline Total & 81 & 100 & 6,0 & 70 & 100 & 6,00 \\
\hline \hline
\end{tabular}

0- sextante hígido, 1- sextante com presença de sangramento em pelo menos um dos dentes, 2- presença de cálculo supra- ou subgengival, 3- bolsa periodontal de 4 a $5 \mathrm{~mm}$ e 4- bolsa periodontal com 6 mm ou mais profunda. 
MENEGHIM, M. de C.; PEREIRA, A. C.; SILVA, F. R. B. Prevalência de cárie radicular e condição periodontal em uma população idosa institucionalizada de Piracicaba - SP. Pesqui Odontol Bras, v. 16, n. 1, p. 50-56, jan./mar. 2002.

TABELA 3 - Estado periodontal, em função do total de sextantes válidos, segundo a faixa etária. Piracicaba, 1998.

\begin{tabular}{c|c|c|c|c}
\hline \hline \multirow{2}{*}{$\begin{array}{c}\text { Estado } \\
\text { periodontal }\end{array}$} & \multicolumn{2}{|c|}{$50-75$ anos } & \multicolumn{2}{c}{ Mais de 75 anos } \\
\cline { 2 - 5 } & $\begin{array}{c}\text { Sextante/ } \\
\text { indivíduo }\end{array}$ & $\%$ & $\begin{array}{c}\text { Sextante/ } \\
\text { indivíduo }\end{array}$ & $\%$ \\
\hline 0 & 0,17 & 30,4 & 0,12 & 66,7 \\
\hline 1 & 0,11 & 19,7 & 0,04 & 22,2 \\
\hline 2 & 0,18 & 32,1 & 0,02 & 11,1 \\
\hline 3 & 0,06 & 10,7 & 0,00 & 0,0 \\
\hline 4 & 0,04 & 7,1 & 0,00 & 0,0 \\
\hline $\begin{array}{c}\text { Total de sex- } \\
\text { tantes válidos }\end{array}$ & 0,56 & 100 & 0,18 & 100 \\
\hline \hline
\end{tabular}

0- sextante hígido, 1- sextante com presença de sangramento em pelo menos um dos dentes, 2- presença de cálculo supra- ou subgengival, 3- bolsa periodontal de 4 a $5 \mathrm{~mm}$ e 4- bolsa periodontal com $6 \mathrm{~mm}$ ou mais profunda.

$22,2 \%$ apresentavam sangramento à sondagem e 0,02 ou $11,1 \%$, apresentavam cálculo na superficie dental (Tabela 3 ).

A relação entre os achados epidemiológicos e a necessidade de tratamento, para os sextantes válidos (Tabela 4), mostra que, para a faixa etária de 50 a 75 anos, 19,7\% dos sextantes necessitavam de instruções de saúde bucal; 42,8\% instrumentação periodontal, enquanto $7,1 \%$ dos sextantes necessitavam de tratamento complexo. Para a faixa etária de mais de 75 anos, a maior porcentagem dos sextantes válidos não necessitava de nenhum tipo de tratamento; 22,2\% necessitavam de instruções de saúde bucal e 11,1\% necessitavam de instrumentação periodontal.

A faixa etária de 50 a 75 anos apresentou 43,9\% ou 83 dentes com retração gengival, sendo que na faixa etária de mais de 75 anos, esse problema é ainda maior, com a participação de 56,1\% ou 106 dentes com retração gengival (Tabelas 5 e 6).

Embora a retração gengival venha sendo considerada como um importante fator predisponente ao desenvolvimento de lesões radiculares ${ }^{4,18}$, analisando-se a percentagem de superficies radiculares afetadas, em relação ao total de dentes remanescentes, demonstrado na Tabela 7 , constata-se que a grande maioria dessas superficies, $96,8 \%$ na faixa etária de 50 a 75 anos de idade e $84,1 \%$ na faixa etária de mais de 75 anos, não desenvolveram lesões radiculares segundo os critérios propostos no estudo.

A maior parte das lesões radiculares diagnosticadas localizava-se nas superficies proximais e
TABELA 4 - Necessidade de tratamento periodontal, de acordo com os sextantes válidos e segundo a faixa etária. Piracicaba, 1998.

\begin{tabular}{c|c|c|c|c}
\hline \hline \multirow{2}{*}{$\begin{array}{c}\text { Necessidade } \\
\text { de tratamento }\end{array}$} & \multicolumn{2}{|c|}{$50-75$ anos } & \multicolumn{2}{c}{ Mais de 75 anos } \\
\cline { 2 - 5 } & $\begin{array}{c}\text { Sextante/ } \\
\text { indivíduo }\end{array}$ & $\%$ & $\begin{array}{c}\text { Sextante/ } \\
\text { indivíduo }\end{array}$ & $\%$ \\
\hline 0 & 0,17 & 30,4 & 0,12 & 66,7 \\
\hline I & 0,11 & 19,7 & 0,04 & 22,2 \\
\hline II & 0,24 & 42,8 & 0,02 & 11,1 \\
\hline III & 0,04 & 7,1 & 0,00 & 0,0 \\
\hline $\begin{array}{c}\text { Total de sex- } \\
\text { tantes válidos }\end{array}$ & 0,56 & 100 & 0,18 & 100 \\
\hline \hline
\end{tabular}

Necessidade de tratamento: 0 - nenhum tratamento; I- instrução de higiene bucal; II- I + instrumentação periodontal; III- I + II + tratamento complexo.

vestibulares. As lesões de cárie proximais foram responsáveis por 58,3\% e 40,0\%; as lesões vestibulares e/ou linguais representaram 41,7\% e $60,0 \%$ nas faixas etárias de 50 a 75 anos e mais de 75 anos, respectivamente.

A percentagem de dentes com retração gengival e afetada por lesões radiculares na faixa etária de mais de 75 anos é maior que a percentagem na faixa etária de 50 a 75 anos de idade. Cerca de 23\% do total de dentes com retração gengival apresentavam também lesões radiculares na faixa etária de 50 a 75 anos de idade. Na faixa etária de mais de 75 anos, essa percentagem atinge 59\%, mostrando que a retração gengival agiu como fator predisponente ao desenvolvimento de lesões de cárie.

\section{DISCUSSÃO}

Na última década, estudos epidemiológicos da doença periodontal têm sido descritos e publicados, sendo a maioria destes estudos baseados no CPITN $^{1,14}$.

O CPITN vem sendo utilizado em pesquisas, na comunidade, por apresentar-se simples e internacionalmente uniforme e, em conseqüência, indicado pela OMS para pesquisa epidemiológica periodontal e por estimar a necessidade de tratamento de grandes grupos populacionais ${ }^{2,14}$.

Em nosso estudo, notamos que o número de sextantes eliminados da amostra aumenta à medida que ocorre um avanço da idade. Tal fato é preocupante, pois mostra a necessidade de uma maior preocupação no desenvolvimento de programas preventivos e curativos aplicados junto às faixas etárias de idade mais avançada, bem como ressal- 
MENEGHIM, M. de C.; PEREIRA, A. C.; SILVA, F. R. B. Prevalência de cárie radicular e condição periodontal em uma população idosa institucionalizada de Piracicaba - SP. Pesqui Odontol Bras, v. 16, n. 1, p. 50-56, jan./mar. 2002.

TABELA 5 - Número e percentagem de dentes com retração gengival em relação ao total de dentes examinados, por faixa etária. Piracicaba, 1998.

\begin{tabular}{l|c|c|c|c|c}
\hline \multirow{2}{*}{ Faixa etária } & \multicolumn{2}{|c|}{ Presença de retração gengival } & \multicolumn{2}{|c|}{ Ausência de retração gengival } & Total \\
\cline { 2 - 6 } & $\mathrm{n}$ & $\%$ & $\mathrm{n}$ & 56,1 & 189 \\
\hline 50-75 anos & 83 & 43,9 & 106 & 41,9 & 55 \\
\hline Mais de 75 anos & 32 & 58,1 & 23 & 52,9 & 244 \\
\hline Total & 115 & 47,1 & 129 & \multicolumn{2}{c}{5} \\
\hline \hline
\end{tabular}

TABELA 6 - Número e percentagem de dentes em relação ao grau de retração gengival e faixa etária. Piracicaba, 1998.

\begin{tabular}{c|c|c|c|c}
\hline \multirow{2}{*}{ Código } & \multicolumn{4}{|c}{ Faixa etária } \\
\cline { 2 - 5 } & \multicolumn{2}{|c|}{$50-75$ anos } & \multicolumn{2}{c}{ Mais de 75 anos } \\
\cline { 2 - 5 } & $\mathrm{n}$ & $\%$ & $\mathrm{n}$ & $\%$ \\
\hline 0 & 106 & 56,1 & 23 & 41,8 \\
\hline I & 37 & 19,6 & 24 & 43,7 \\
\hline II & 27 & 14,3 & 6 & 10,9 \\
\hline III & 19 & 10,0 & 2 & 3,6 \\
\hline IV & 0 & 0,0 & 0 & 0,0 \\
\hline Total & 189 & 100 & 55 & 100 \\
\hline \hline
\end{tabular}

Retração gengival: 0- maior ou igual a 0 e menor ou igual a $0,5 \mathrm{~mm}$; I- maior que $0,5 \mathrm{~mm}$ e menor ou igual a $3 \mathrm{~mm}$; II- maior que 3 e menor ou igual a $5 \mathrm{~mm}$; III- maior que $5 \mathrm{~mm}$ e menor ou igual a $7 \mathrm{~mm}$; IV- maior que $7 \mathrm{~mm}$.

ta a necessidade de um direcionamento das pesquisas para o paciente idoso.

Estudo similar realizado por PEREIRA et $a .^{15}$ (1996), realizado em pacientes usuários de um centro de saúde, portanto não institucionalizados, mostrou 4,29 sextantes eliminados da amostra para a faixa etária entre 60 e 70 anos e 4,71 para a faixa etária de mais de 70 anos, confirmando o pequeno número de dentes presentes em cada sextante.

Como a faixa etária de mais de 75 anos apresenta um maior número de sextantes eliminados e, portanto, um maior número de dentes extraídos que a faixa etária entre 50 e 75 anos, a necessidade de tratamento periodontal para os sextantes remanescentes é maior na segunda faixa etária. Para a faixa etária entre 50 e 75 anos, apenas $30,4 \%$ não necessitavam de nenhum tratamento periodontal, enquanto $42,8 \%$ necessitavam de controle de placa e instrumentação periodontal. Dessa forma, é importante salientar que os resultados obtidos são semelhantes àqueles encontrados por OKAWA et al. ${ }^{12}$ (1993), ROSA ${ }^{16}$ (1992) e FURE ${ }^{5}$ (1997), em que os
TABELA 7 - Número e percentagem de lesões com envolvimento radicular para as faixas etárias de 50 a 75 anos e com mais de 75 anos de idade. Piracicaba, 1998.

\begin{tabular}{c|c|c|c|c}
\hline \hline Código & $50-75$ anos & $\%$ & Mais de 75 anos & $\%$ \\
\hline 0 & 732 & 96,8 & 185 & 84,1 \\
\hline LI & 18 & 2,4 & 17 & 7,7 \\
\hline LII & 3 & 0,4 & 3 & 1,4 \\
\hline LIII & 0 & 0,0 & 0 & 0,0 \\
\hline LIV & 3 & 0,4 & 15 & 6,8 \\
\hline Faces & 756 & 100 & 220 & 100 \\
\hline \hline
\end{tabular}

Lesões radiculares: 0- ausência de lesão; LI- lesão inativa de cárie radicular; LII- lesão ativa de cárie radicular; LIII- obturação; LIV- erosão/abrasão.

procedimentos simples de tratamento, como instrução de higiene bucal e remoção de tártaro, podem ser delegados ao pessoal técnico em higiene dental (THD) ficando os procedimentos mais complexos, que compreendem tratamento de bolsas profundas, com o profissional especializado.

Esse aspecto é importante na questão do planejamento do serviço odontológico, pois medidas de promoção da saúde podem ser tomadas com uma relação custo-benefício favorável.

Apesar de se apresentar simples e uniforme e, em conseqüência, recomendado pela OMS para pesquisa epidemiológica, o CPITN não é um estimador das condições periodontais aceito como unanimidade pelos pesquisadores ${ }^{2,9}$, que citam a fragilidade do índice CPITN como ferramenta epidemiológica, argumentando que as marcações periciais podem subestimar a prevalência das bolsas periodontais, distorcendo as estimativas da prevalência e severidade da doença periodontal.

Um outro problema que pode ser discutido é a reprodutividade dos indices periodontais. MOJON et $a .^{10}$ (1996), estudando esta reprodutividade mostraram, através da estatística Kappa, que a não-concordância ocorre, principalmente, para sangramento e bolsas rasas. 
MENEGHIM, M. de C.; PEREIRA, A. C.; SILVA, F. R. B. Prevalência de cárie radicular e condição periodontal em uma população idosa institucionalizada de Piracicaba - SP. Pesqui Odontol Bras, v. 16, n. 1, p. 50-56, jan./mar. 2002.

Em relação à cárie de raiz, deve-se levar em conta um aspecto importante ao comparar os resultados desta pesquisa com resultados de outros autores, que se referem ao fato de os critérios para a lesão de cárie radicular variarem bastante.

BECK $^{3}$ (1993); KATZ ${ }^{8}$ (1990) salientam que os critérios para o diagnóstico da lesão cariosa variam bastante de um estudo para outro, podendo-se levar em consideração questões como cor, textura ou profundidade e as prevalências serem apresentadas como número médio de lesões, de dentes ou ainda de superficies atacadas.

A OMS $^{14}$ (1999), em seu manual de levantamento básico em saúde bucal, divulgou códigos e critérios de classificação para o exame de superficie radicular, que certamente muito irá contribuir para a uniformidade das pesquisas epidemiológicas.

A presença de lesões radiculares tem sido a causa de grande preocupação nessa faixa etária. Para a faixa etária de mais de 75 anos, pode-se encontrar uma prevalência de $9,1 \%$ de coroas cariadas e nenhuma superficie radicular afetada. WINN et al. ${ }^{20}(1996)$ encontraram, na mesma faixa etária, uma prevalência de $6,8 \%$ para os componentes cariado e restaurado. A ausência de dentes submetidos a tratamento restaurador revela a total ausência de programas curativos. Pelo menos $70 \%$ das pessoas com faixa etária de mais de 75 anos apresentava pelo menos uma experiência de cárie. Resultado similar pode se observado até mesmo em países desenvolvidos, com uma prevalência de $55,9 \%$ de pessoas com presença de dentes cariados e/ou obturados ${ }^{20}$, sem levar em conta a presença de lesões radiculares de erosão e abrasão muito comuns nessa faixa etária e demonstradas no estudo realizado.

A grande prevalência de pessoas com histórico de lesões radiculares mostra a ausência de programas curativos e o despreparo dos odontólogos frente ao desenvolvimento e avanço de tais tipos de lesões que se tornam cada vez mais freqüentes à medida que o número de dentes remanescentes na cavidade bucal é cada vez maior.

Estes dados reforçam a premissa que a Gerodontologia deveria ser vista de uma forma mais critica, fazendo parte integrante do planejamento de serviço em Odontologia, uma vez que se percebe a necessidade que estas pessoas apresentam na obtenção de orientação em saúde bucal e acesso a cuidados odontológicos básicos. Dessa forma, como relatado por WERNER et al. ${ }^{19}$ (1998), o atendimento ao idoso passa a ter maior relevância, pois uma Odontologia de promoção de saúde deve levar em conta aspectos relacionados à habilidade funcional do paciente, comunicação, relacionamento com a família, doenças associadas ao envelhecimento, entre outras coisas. De acordo com as tendências de envelhecimento da população, é importante a discussão do desenvolvimento de um currículo específico, na graduação e pós-graduação, para a Odontologia geriátrica, dessa forma preparando o cirurgião-dentista para esta nova realidade.

Como profissionais preocupados com uma Odontologia de promoção de saúde, devemos elaborar programas não só baseados nas necessidades de tratamento e métodos de prevenção, mas que visem também à educação do paciente na aquisição de hábitos de higiene bucal.

\section{CONCLUSÕES}

Constatou-se que:

1. apesar de ocorrer uma grande prevalência de problemas periodontais, esses são, em sua maior parte, de baixa complexidade e passiveis de serem delegados ao profissional técnico (THD);

2. observou-se elevada porcentagem de lesões de cárie radicular, indicando que o cirurgião-dentista deve estar atento a tal problema, procurando atualizar-se neste assunto com referência ao diagnóstico, prevenção e terapêutica.

MENEGHIM, M. de C.; PEREIRA, A. C.; SILVA, F. R. B. Prevalence of root caries and periodontal conditions in an elderly institutionalized population from Piracicaba - SP. Pesqui Odontol Bras, v. 16, n. 1, p. 50-56, jan./mar. 2002.

Preventive and invasive dental procedures have improved in the last two decades, leading to a greater longevity of teeth. As a result, teeth are more susceptible to periodontal diseases and, consequently, to root caries. The purpose of this study was to verify the prevalence of both periodontal diseases and root caries in an institutionalized population from Piracicaba - SP, Brazil. In this study, 151 subjects, aging 54 to 93 years, were examined. The sample was divided into two groups: a group of subjects aging 50 to 75 years and a group of subjects over 75 years old. The sextants were examined and classified as to periodontal health, periodontal treatment needs, prevalence of gingival recession and root lesions. In conclusion, a high prevalence of root caries was verified. That indicates an increase in the susceptibility to these lesions, which is associated with the aging process.

UNITERMS: Geriatric dentistry; Epidemiology; Root caries. 
MENEGHIM, M. de C.; PEREIRA, A. C.; SILVA, F. R. B. Prevalência de cárie radicular e condição periodontal em uma população idosa institucionalizada de Piracicaba - SP. Pesqui Odontol Bras, v. 16, n. 1, p. 50-56, jan./mar. 2002.

\section{REFERÊNCIAS BIBLIOGRÁFICAS}

1. AINAMO, J. Development of the World Health Organization (WHO) Community Periodontal Index of Treatment Needs (CPITN). Int Dent J, v. 32, n. 3, p. 281-291, Sept. 1982.

2. BAELUM, V.; MANJI, F.; WANZALA, P. et al. Relationship between CPITN and periodontal attachment loss findings in an adult population. J Clin Periodontol, v. 22, n. 2, p. 146-152, Feb. 1995.

3. BECK, J. D. The epidemiology of root surface caries: North American studies. Adv Dent Res, v. 7, n. 1, p. 42-51, Sept. 1993.

4. FEJERSKOV, O. Recent advancements in the treatment of root surface caries. Int Dent J, v. 44, p. 139-144, Apr. 1994.

5. FURE, S. Five-year incidence of coronal and root caries in 60-, 70- and 80-year-old Swedish individuals. Caries Res, v. 31, n. 3, p. 249-258, May/June 1997.

6. INSTITUTO BRASILEIRO DE GEOGRAFIA E ESTATÍSTICA. Brasil está mais velho e violento, diz IBGE. Brasil On Line, 10 mar. 1999, Brasil, p. 1 de 2.

7. JANCZUC, $Z$. Oral health of Polish children and WHO/FDI goals for the year 2000. Community Dent Oral Epidemiol, v. 17, n. 2, p. 75, Apr. 1989.

8. KATZ, R. V. Clinical signs of roots caries: measurement issues from an epidemiologic perspective. J Dent Res, v. 69, n. 5, p. 1211-1215, May 1990.

9. MILlER, N. A.; BENAMGHAR, L.; ROLAND, E. et al. An analysis of the CPITN. Studies on adults in France. III partial examinations versus full mouth examinations. Community Dent Health, v. 7, n. 3, p. 249-253, 1990.

10. MOJON, P.; CHUNG, J. P.; FAVRE, P. et al. Examiner agreement on periodontal indices during dental surveys of elders. J Clin Periodontol, v. 23, n. 1, p. 56-59, Jan. 1996.

11. NEWBRUN, E. et al. Root caries. CDA J, v. 12, n. 12, p. 68-73, Jan. 1984.

12. OKAWA, Y.; SUGIHARA, N.; MAKI,Y. et al. Prevalence of root caries in a Japanese adult population aged 20-59 years. Bull Tokyo Dent Coll, v. 34, n. 3, p. 107-113, Aug. 1993

13. OPPERMANN, R. V.; ROSING, C. K. Prevenção e tratamento das doenças periodontais In: ABOPREV. Promoção de saúde bucal. São Paulo : Artes Médicas, 1997. Cap. 12, p. 255-282.

14. ORGANIZAÇÃO MUNDIAL DA SAÚDE. Levantamento epidemiológico básico de saúde bucal, manual de instruções. 4. ed. São Paulo : Santos, 1999. 66 p.

15. PEREIRA, A. C.; SILVA, S. R. C.; CASTELLANOS, R. A. et al. Oral health and periodontal status in Brazilian elderly. Braz Dent J, v. 7, n. 2, p. 97-102, 1996.

16. ROSA, A. G. F. Condições de saúde bucal em pessoas de 60 anos ou mais no Município de São Paulo (Brasil). Rev Saúde Públ, v. 26, n. 3, p. 155-160, Jun. 1992.

17. TOLEDO, J. R. Brasil sobe 6 posições em lista da ONU IDH Folha de S. Paulo, São Paulo, 9 set. 1998, Brasil, p. 8.

18. VEHKALAHTI, M.; PAUNIO, I. Association between root caries occurrence and periodontal state. Caries Res, v. 28, n. 4, p. 301-306, July/Aug. 1994.

19. WERNER, C. W.; SAUNDERS, M. J.; PAUNOVICH, E. et al. Odontologia geriátrica. Rev Fac Odontol Lins, v. 11, n. 1, p. 62-70, jan./jun. 1998.

20. WINN, D. M.; BRUNELLE, J. A.; SELWITZ, R. H. et al. Coronal and root caries in the dentition of adults in the United States, 1988-1991. J Dent Res, v. 75, Spec. Iss., p. 642-651, Feb. 1996.
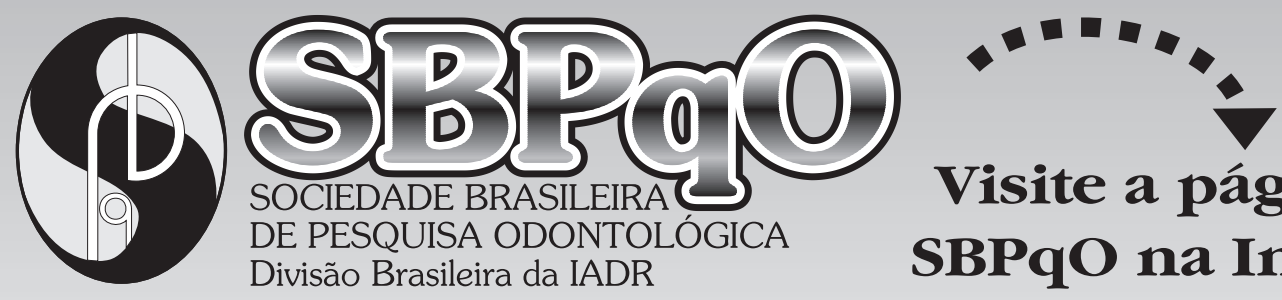

Visite a página da

Divisão Brasileira da IADR

SBPqO na Internet:

http://www.sbpqo.org.br

\author{
SBPqO - Av. Professor Lineu Prestes, 2.227 \\ CEP 05508-900 - Cidade Universitária - São Paulo - SP \\ Tel./Fax: (0**11) 3091-7855 - E-mail: sbpqo@sbpqo.org.br
}

